\title{
-Original-
}

\section{The characteristics of a porcine mitral regurgitation model}

\author{
Bo $\mathrm{LI}^{1)}$, Yongchun $\mathrm{CUI}^{1)}$, Dong $\mathrm{ZHANG}^{2)}$, Xiaokang $\mathrm{LUO}^{1)}$, Fuliang $\mathrm{LUO}^{1)}$, \\ Bin $\mathrm{LI}^{1)}$, and Yue TANG ${ }^{1)}$ \\ 1) Animal Experimental Centre, Beijing Key Laboratory of Preclinical Research and Evaluation for Cardiovascular \\ Implant Materials, State Key Laboratory of Cardiovascular Disease, Fuwai Hospital, National Centre for \\ Cardiovascular Disease, Chinese Academy of Medical Sciences and Peking Union Medical College, No.167 North \\ lishi Road, Xicheng District, Beijing 100037, China \\ ${ }^{2)}$ Department of Cardiovascular surgery, Beijing Jishuitan Hospital, No. 31 Xinjiekou East Street, Xicheng District, \\ Beijing 100035, China
}

\begin{abstract}
The porcine mitral regurgitation (MR) model is a common cardiovascular animal model. Standardized manufacturing processes can improve the uniformity and success rate of the model, and systematic research can evaluate its potential use. In this study, 17 pigs were divided into an experimental group $(n=11)$ and a control group $(n=6)$. We used a homemade retractor to cut the mitral chordae via the left atrial appendage to establish a model of MR; the control group underwent a sham surgery. The model animals were followed for 30 months after the surgery. Enlargement and fibrosis of the left atrium were significant in the experimental group compared with those in the control group, and left atrial systolic function decreased significantly. In addition, model animals showed preserved left ventricular systolic function. There were no differences in left atrial potential or left ventricular myocardial fibrosis between the two groups. Atrial fibrillation susceptibility in the experimental group was higher than that in the control group. Our method enables the simple and effective production of a MR model with severe reflux that can be used for pathophysiological studies of MR, as well as for the development of preclinical surgical instruments and their evaluation. This model could also be used to study atrial fibrillation and myocardial fibrosis but is not suitable for studies of heart failure. Key words: animal model, atrial fibrillation susceptibility, mitral regurgitation, myocardial fibrosis, preclinical evaluation
\end{abstract}

\section{Introduction}

Mitral regurgitation (MR) is the most common valvular disease, and the number of affected patients will continue to increase as the population ages [23, 24]. MR is divided into two categories: (1) organic MR (primary), which is due to abnormalities of the mitral leaflets and subvalvular apparatus; and (2) functional MR (secondary), which is caused by enlargement of the annulus and displacement of the papillary muscles but normal mitral leaflets and an unaffected subvalvular apparatus. Although progress has been made in terms of treatment, the mechanism of MR, especially functional MR, is unclear $[4,37]$. Further studies of the MR animal mod-

(Received 22 March 2018 / Accepted 1 May 2018 / Published online in J-STAGE 22 May 2018)

Address corresponding: Y. Tang, Animal Experimental Centre, Beijing Key Laboratory of Preclinical Research and Evaluation for Cardiovascular Implant Materials, State Key Laboratory of Cardiovascular Disease, Fuwai Hospital, National Centre for Cardiovascular Disease, Chinese Academy of Medical Sciences and Peking Union Medical College, No.167 North lishi Road, Xicheng District, Beijing 100037, China

Supplementary Figure: refer to J-STAGE: https://www.jstage.jst.go.jp/browse/expanim

(c) $\$($ This is an open-access article distributed under the terms of the Creative Commons Attribution Non-Commercial No Derivatives (by-nc-nd) License <http://creativecommons.org/licenses/by-nc-nd/4.0/>.

(C)2018 Japanese Association for Laboratory Animal Science 
els could provide a better understanding of the disease. In addition, the increasing variety of operating equipment used to treat MR $[9,35,41]$ and related diseases [21] necessitates pre-clinical evaluations in animal models. Therefore, there is a great demand for an animal model of MR.

An effective large animal model of MR is an important tool for cardiovascular research because it would not only meet the needs of basic research but could also be used for the pre-clinical evaluation of surgical instruments. There are many methods for establishing a MR model, but the available models have been unsatisfactory. Specifically, difficulties associated with model generation, such as poor uniformity, high mortality, complications and insufficient reflux, cause studies using these models to be time-consuming and labourious. In addition, studies involving large-animal MR models are short-term and occasionally only acute. The outcomes of these models over an extended period of time and whether the information they provide could be useful in the study of other diseases or pathological processes remain poorly understood.

The purpose of this study was to establish a porcine MR model with good uniformity, a high success rate, few complications and severe reflux. Additionally, a long-time and systematic study was conducted using the porcine MR model to determine its potential use in cardiovascular disease.

\section{Materials and Methods}

\section{Animals}

The experiments were conducted using 10-month-old male Guizhou miniature pigs weighing $25-35 \mathrm{~kg}$, which were provided by the animal experimental centre of $\mathrm{Fu}$ wai Hospital. Before and after the operation, the animals were maintained in a normal environment, including a $12 \mathrm{~h} \mathrm{light/dark} \mathrm{cycle,} \mathrm{room} \mathrm{temperature} \mathrm{at} 25^{\circ} \mathrm{C}$. Experimental animals were free to access drinking water and fed with commercial maintenance feed twice daily. All of the animal procedures were approved by the animal welfare ethics committee of Fuwai Hospital of Peking Union Medical College, and all of the experiments were conducted in accordance with National Regulations on the Administration of Laboratory Animals.

General anaesthesia with Pentobarbital (30-50 mg/ $\mathrm{kg})$ and midazolam $(0.1-0.5 \mathrm{mg} / \mathrm{kg})$ was administered intramuscularly. The pigs were intubated with a cuffed endotracheal tube, the tidal volume was $12 \mathrm{ml} / \mathrm{kg}$, and the oxygen concentration was $100 \%$. Maintenance of anesthesia were performed by intravenous injection of fentanyl through auricular vein, $2-10 \mathrm{mg} / \mathrm{kg}$ was injected for the first time and then superadded according to the level of anaesthesia. The animal model was established as described previously [7], but without ameroid ring implantation. The pig was placed in the right lateral position with the left chest exposed. The whole process required the use of sterile techniques. A left intercostal thoracotomy between the third and fourth ribs was created, and the pericardium was opened to expose the heart. A purse string suture was made in the left atrial appendage. Through this opening, an $8 \mathrm{~F}$ sheath was inserted into the left atrium at a depth of $2.5-3.0 \mathrm{~cm}$ to check and record the pressure of the left atrium, which ranged from approximately $6 \mathrm{mmHg}$ to $8 \mathrm{mmHg}$ average pressure. We then continued to insert the sheath into the right ventricle and determined the front-end position of the sheath according to the pressure. The average left ventricular pressure ranged from approximately 35 $\mathrm{mmHg}$ to $45 \mathrm{mmHg}$, and the pulse pressure increased. With the aid of epicardial ultrasound monitoring, a homemade retractor was inserted into the left ventricle via the sheath, and the chordae tendineae of the $\mathrm{P} 2$ and P3 area of the posterior mitral leaflet were hooked and cut (Fig. 1). The sheath was withdrawn to the left atrium, and the left atrial pressure was measured after $15 \mathrm{~min}$, resulting in an increase of at least $10 \mathrm{mmHg}$. If the left atrial pressure failed to increase by $10 \mathrm{mmHg}$, we repeated the previous procedure until the pressure met this requirement (Supplementary Fig. 1). In the control group, pigs were treated with the same surgical procedure as in the experimental group but did not undergo injury of the mitral valve. The thorax was then closed, and pethidine hydrochloride $(1 \mathrm{mg} / \mathrm{kg}$, im, bid) was administered for 2 days, cephalexin $(15 \mathrm{mg} / \mathrm{kg}$, im, bid) for 3 days and furosemide (10 mg, im, bid) for 7 days. After surgery, the pigs continued to receive digoxin, furosemide, spironolactone, and potassium citrate granules orally for 1 month. Daily auscultation was necessary to assess the presence of pulmonary oedema.

\section{Electrocardiogram}

Each pig was examined via a twelve-lead electrocardiography (GE medical systems, Boston, USA) before surgery and sampling. The same anaesthetic method as described above was used for these procedures. The hair 

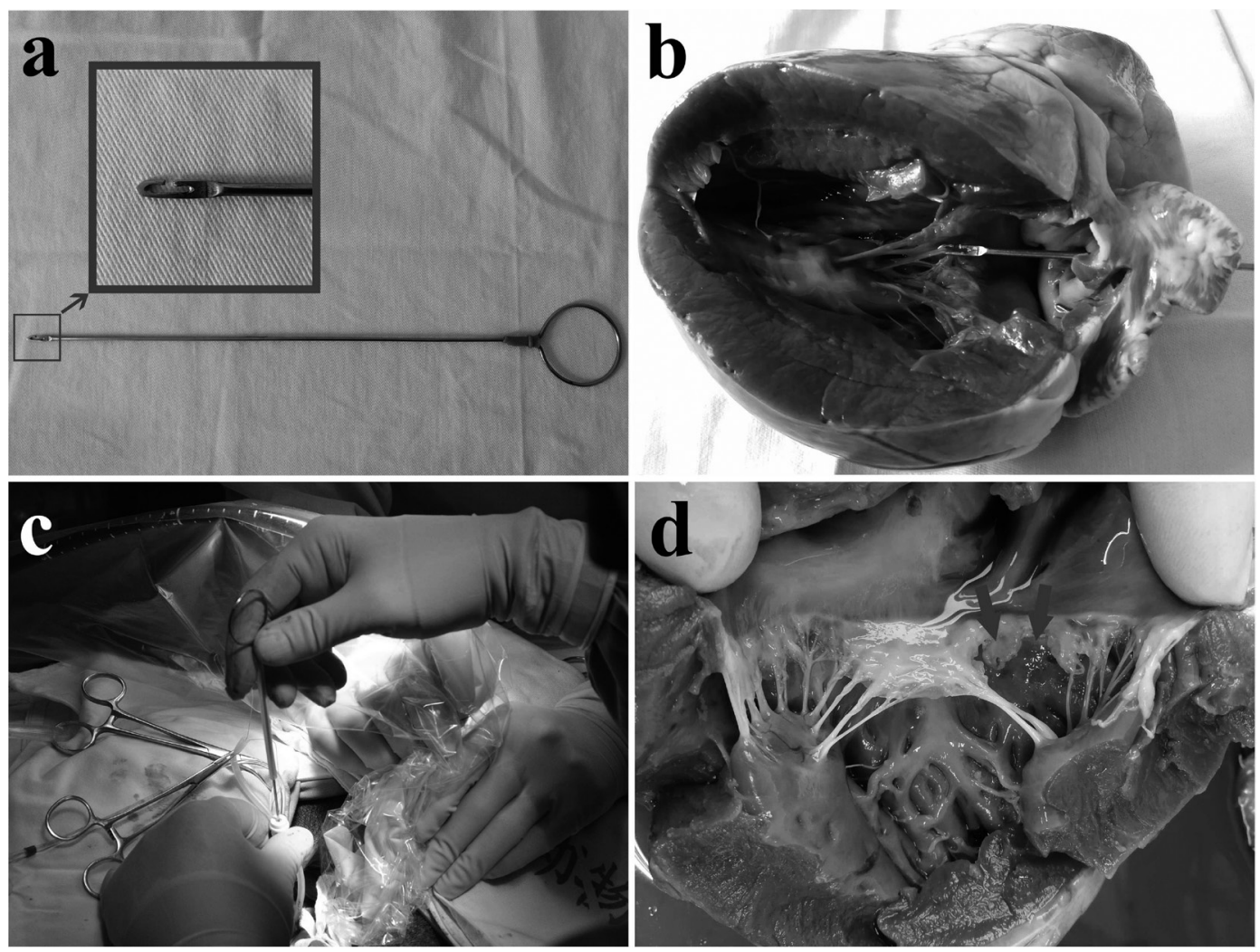

Fig. 1. The structure and working principle of the homemade retractor. a. The structure of the homemade retractor; b. Working principle of the retractor in isolated Heart; c. The use of retractor during surgery; d. Mitral valve leaflets damaged by the retractor. The arrows indicate the mitral valve leaflet with a broken tendon.

of the left chest and leg was removed after anaesthesia, and the electrocardiograms were recorded as previously described [9]. Three standard bipolar limb lead electrodes were placed on juxta-articular regions of the pig, electrode V1-V6 chest lead electrode plates were attached to the chest; V1 was placed at the right edge of the sternum in the fourth intercostal space; V2 was placed at the left edge of the sternum in the fourth intercostal space; V4 was placed at the intersection of the left midclavicular line and the fifth intercostal space; V3 was placed at the midpoint of the connection of V2 and V4; V5 was placed at the anterior axillary line and in the same horizontal line as V4; and V6 was placed at the left midaxillary line and in the same horizontal line as V4 and V5. The electrode was then connected to the electrocardiograph to record the ECG. The correction voltage was $1 \mathrm{mv}=10 \mathrm{~mm}$, and the paper speed was $25 \mathrm{~mm} / \mathrm{s}$. The ECG was interpreted by a cardiologist with extensive experience analysing ECGs.

\section{Echocardiography}

Echocardiographic studies were performed using a high-quality echocardiograph (Vivid 7, GE, Waukesha, Wisconsin) equipped with a $2.5-\mathrm{MHz}$ transducer. Measurements of the degree of MR, left atrial maximal volume (LAV max), left atrial minimal volume (LAV min), left atrial ejection fraction (LAEF), left ventricular enddiastolic volume (LVEDV), left ventricular end-systolic volume (LVESV), and left ventricular ejection fraction (LVEF) were performed in accordance with the current recommendations of the American Society of Echocardiography. We used the ratio of the regurgitation area to left atrial area (RA/LAA) to evaluate the degree of regurgitation: less than $20 \%$ indicated mild regurgitation, between $20 \%$ and $40 \%$ indicated moderate regurgitation, and greater than $40 \%$ indicated severe regurgitation. LAV max and LAV min were calculated from the apical four-chamber and two-chamber views using the modified Simpson biplane method. The LAV max (defined as the volume immediately prior to mitral valve opening) and the LAV min (defined as the volume immediately after 
mitral closure) were recorded, followed by the derivation of LAEF, as follows: (LAV max-LAV min)/LAV $\max \times 100 \%$ [29]. The LVEDV and LVESV were calculated from the left ventricular long axis view using Simpson's biplane method. The LVEDV (defined as the maximum volume of left ventricular diastolic phase) and the LVESV (defined as minimum volume of left ventricle systolic phase) were recorded, followed by derivation of the LVEF, as follows: (LVEDV-LVESV)/ LVEDV $\times 100 \%$. Both transthoracic and epicardial ultrasound echocardiography were performed by experienced accredited sonographers and physicians. A transthoracic ultrasound was performed before the operation to eliminate pigs with structural heart disease. Intraoperative epicardial echocardiography was accomplished using an oesophageal ultrasound probe to monitor the severity of MR during the operation. After the operation, the pigs were examined by transthoracic ultrasound at 1 month, 6 months, 12 months, 18 months, 24 months, and 30 months for review until the end of the experiment.

\section{Three-dimensional electrophysiological mapping}

Before the end of the experiment, electrical mapping of the left atrium was performed using a three-dimensional electrophysiological mapping system (Ensite3000, St Jude Medical, Inc., St. Paul, MN). The specific electrode was inserted into the left atrium via the femoral vein approach, with puncture of the atrial septum. The electrode catheter was inserted along the left atrium endocardium under guidance from the X-ray image and intracardiac electrogram. The three-dimensional geometric configuration of the endocardium in the left atrium at the end of diastole was established, and the maximum amplitude of the electrogram in the endocardium was collected. The amplitude was indicated by red, yellow, green, blue, and purple, with red representing the lowest amplitude and purple the highest. The left atrial voltage map revealed the difference between the low-voltage and normal myocardium.

\section{Histological analyses}

The left atrium was removed from the heart and fixed in $10 \%$ formalin for $48 \mathrm{~h}$. Specimens measuring approximately $1.0 \mathrm{~cm} \times 0.5 \mathrm{~cm}$ were obtained from the left posterior wall of left atrium central and left ventricular anterior wall. Epicardial connective tissue was removed to avoid overestimating the degree of fibrosis. Sirius Red staining was performed using a kit from LEAGENE
(Beijing, China), according to the manufacturer's protocol. Collagen I was stained red and orange, collagen III was stained green, and cell nuclei were stained blue. The degree of myocardial fibrosis was measured and quantified by Sirius Red staining using Image-Pro Plus 6.0. For each specimen, 10 fields were randomly selected, and the proportion of fibrotic areas were statistically analysed. The collagen volume fraction $(\mathrm{CVF})$ was calculated as follows: $\mathrm{CVF}(\%)=$ (total area of collagens /total area of image) $\times 100 \%$.

\section{Atrial fibrillation susceptibility test}

All of the laboratory animals, including those in the experimental group and the control group, were tested for susceptibility to atrial fibrillation. The pigs were anaesthetized as previously described, and the chest was opened via midline sternotomy. The stimulating electrode was connected to the right auricle. Atrial fibrillation susceptibility was tested by burst pacing with $600 \mathrm{bpm}$ at the LA site, and $8 \times 6 \mathrm{~s}$ bursts were applied. The duration of atrial fibrillation was measured in each animal. If an episode lasted longer than $180 \mathrm{~s}$ after any stimulus, atrial fibrillation susceptibility was considered positive. After AF vulnerability testing, experimental animals were administered euthanasia by intravenous injection of $10 \%$ potassium chloride injection $(0.5-1 \mathrm{ml} / \mathrm{kg}$ ) under anaesthesia, then tissues were harvested for histology.

\section{Statistical analysis}

All values were analysed with SPSS 24.0 and expressed as the mean $\pm \mathrm{SE}$ of the mean. A single-sample $t$-test was used to compare the mean of the sample with the known population mean. An independent-samples $t$-test was used to compare two sample means. Two-way ANOVA with repeated measures was conducted to examine differences between the groups and between measurement times. An independent-samples $t$-test was used for intergroup comparisons, and a paired-samples $t$-test was used for intragroup comparisons. The $\chi^{2}$ test was used to examine the statistical significance of the positive rate of the atrial fibrillation susceptibility test between the two groups. $P$-values less than 0.05 were considered statistically significant. GraphPad Prism 7 software was used for the statistical analyses.

\section{Data availability statement}

The datasets generated during and/or analysed during the current study are available from the corresponding 

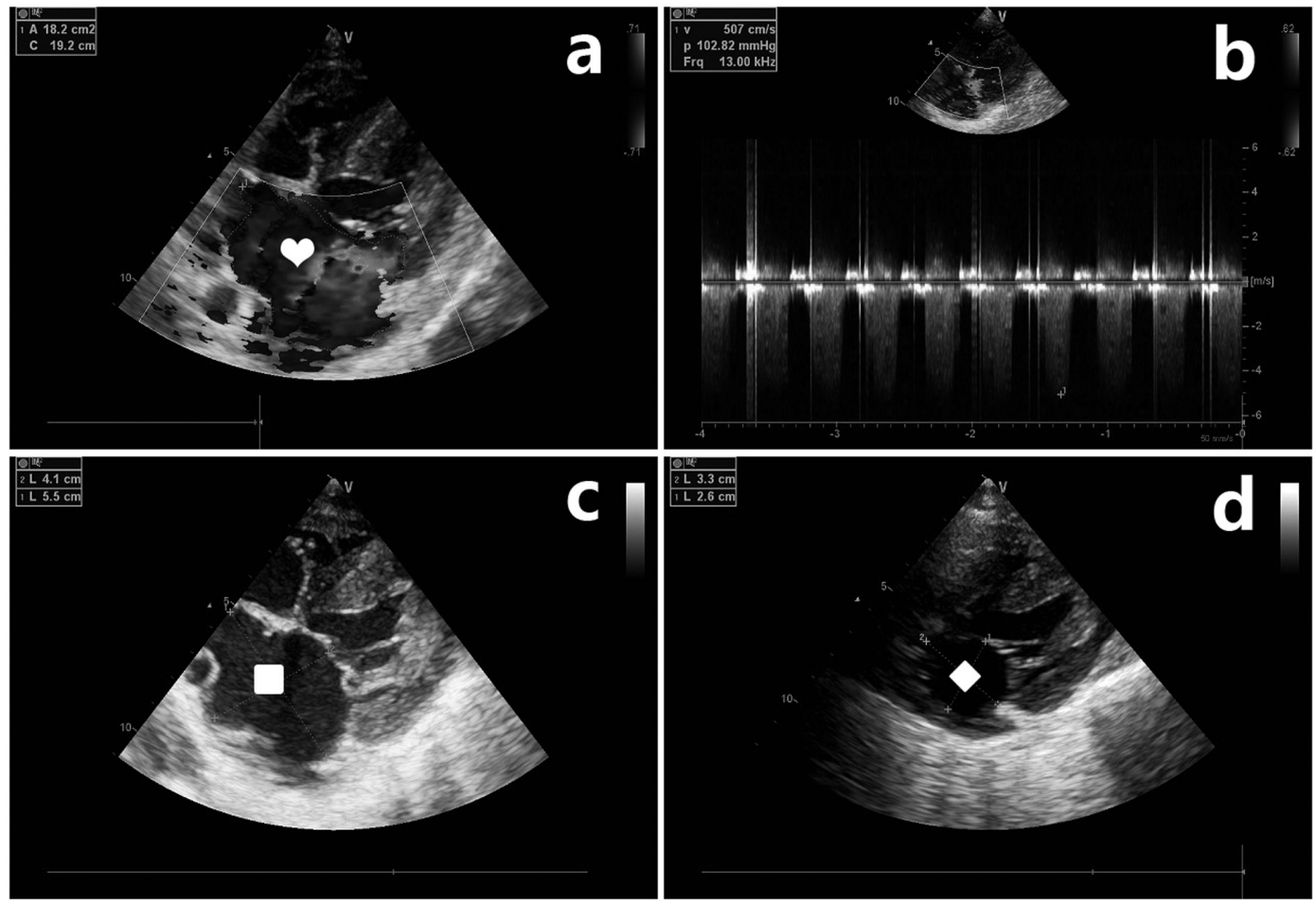

Fig. 2. Ultrasound cardiogram of the pig. a: The apical four chamber view showing blood flowing through the mitral valve and flowing back into the left atrium. Heart: The blue area is the regurgitant beam. b: Continuous-wave Doppler at the level of the mitral valve showing the holosystolic regurgitant flow. c: The anteroposterior diameter and left and right diameter of the left atrium increased significantly in the mitral regurgitation group ( $4.1 \mathrm{~cm}$ and $5.5 \mathrm{~cm}$, respectively). Square: Enlarged left atrium. d: The anteroposterior diameter and left and right diameter of the left atrium in the control group were normal $(2.6 \mathrm{~cm}$ and $3.3 \mathrm{~cm}$, respectively). Diamond: Normal left atrium.

author on reasonable request.

\section{Results}

\section{Surgical results}

In this study, 11 pigs underwent MR surgery. 2-3 times cutting were usually required to meet our requirements during the operation, and post-operative cardiac anatomy showed that the lesion site was located in the P2 and P3 area of the posterior mitral leaflet where we are expected (Fig. 1). One of the pigs had a left atrial posterior wall rupture and bled during the operation, which was stopped by suturing. Due to excessive bleeding and MR, the pig died $4 \mathrm{~h}$ after the operation. In the postoperative review, the degree of MR in one pig was not sufficient, and the pig was excluded from the analysis. At the end of the experiment, another pig developed ventricular fibrillation during anaesthesia for specimen collection and died after rescue. The other 8 pigs were confirmed by echocardiography as having severe MR (Fig. $2 a$ and b). No pigs experienced postoperative heart failure, pulmonary oedema, wound infection, pulmonary infection, or other complications. The operative mortality rate was $9 \%$, and the success rate of the model was $82 \%$.

\section{Electrocardiogram}

The preoperative ECG showed that the $\mathrm{P}$ wave width of the experimental group was similar to that of the control group. The $\mathrm{P}$ wave width in the experimental group increased gradually, and some of the animals showed double-cusp type changes (Fig. 3). However, we did not observe signs of left ventricular hypertrophy or enlargement, such as an increased QRS wave group voltage, left axis deviation, or and ST-T changes.

\section{Echocardiography}

The degree of MR: In the experimental group, all of the pigs were confirmed by ultrasonography as exhibiting severe MR (RA/LAA $>40 \%)$ at different times, and the RA/LAA values at $1,6,12,18,24$, and 30 months 


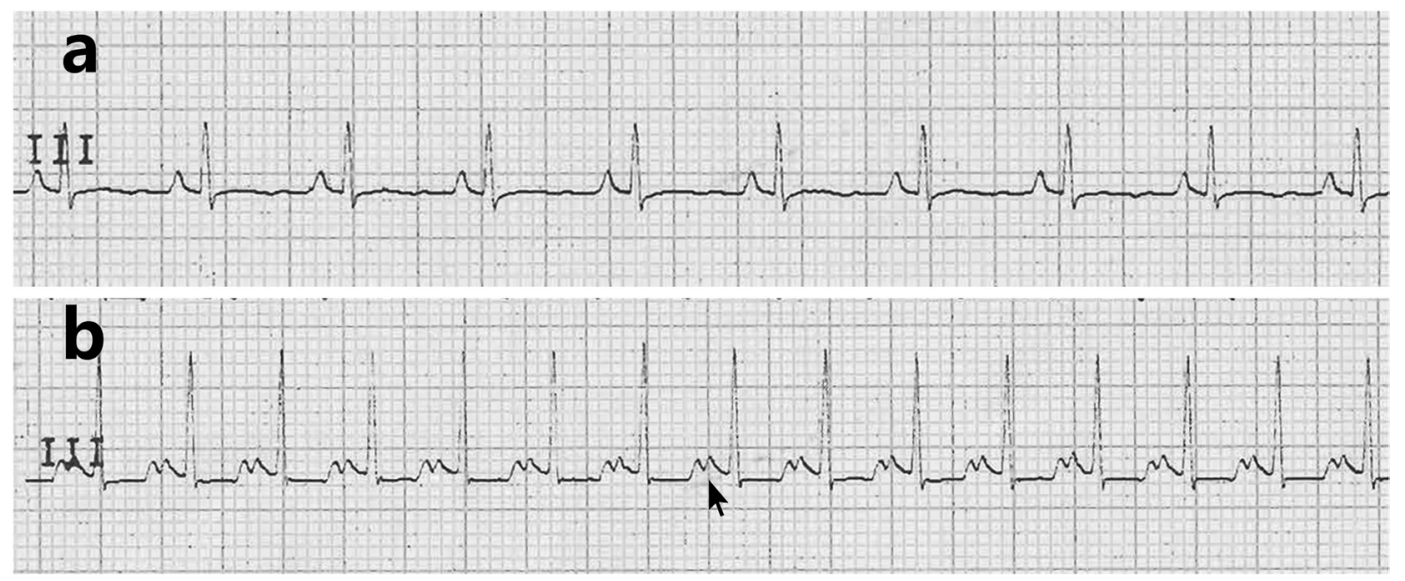

Fig. 3. The ECGs of the experimental group and control group were compared at 30 months after the operation. a: The height and width of the $\mathrm{P}$ wave were normal in the experimental group based on the electrocardiogram b: Electrocardiography in the experimental group showed that the $\mathrm{P}$ wave broadened and exhibited a doublecusp type, indicated by the arrow.

after the operation were $54.3 \pm 3.5 \%, 46.8 \pm 1.6 \%, 51.8$ $\pm 4.3 \%, 47.8 \pm 1.4 \%, 46.9 \pm 1.0 \%$, and $47.0 \pm 1.5 \%$, respectively. There were no cases of mitral stenosis or regurgitation in the control group during the experiment.

Left atrial volume and ejection fraction: Two-way ANOVA with repeated measures found significant intergroup, intragroup and interactional differences in the LAV-max, LAV-min, and LAEF (Table 1).

An independent-samples $t$-test found no intergroup difference in the LAV-max, LAV-min, or LAEF before the operation $(P=0.145, P=0.138, P=0.824)$. The LAV max and LAV min of the experimental group were higher than those in the control group at each time point after the operation. There was no difference in the LAEF between the two groups in the early post operation $((P=0.221, P=0.053, P=0.070$ at 1,6 , and 12 months after the operation). However, at late post-operation time points, the LAEF of the experimental group was lower than that of the control group $(P=0.002, P=0.006$, $P=0.001$ at 18,24 , and 30 months after the operation) (Table 1).

In the control group, there was no intragroup difference in the LAV-max, LAV-min, LAEF. In the experimental group, LAV max and LAV min increased from $12.1 \pm 1.5 \mathrm{ml}$ and $4.8 \pm 0.5 \mathrm{ml}$, respectively, before the surgery to $25.8 \pm 1.9 \mathrm{ml}(P<0.001)$ and $11.5 \pm 1.2 \mathrm{ml}$ $(P=0.004)$ at 1 month, $31.0 \pm 1.9 \mathrm{ml}(P<0.001)$ and 14.9 $\pm 1.4 \mathrm{ml}(P=0.001)$ at 6 months, $30.0 \pm 2.0 \mathrm{ml}(P<0.001)$ and $15.6 \pm 1.3 \mathrm{ml}(P<0.001)$ at 12 months, $31.1 \pm 1.4 \mathrm{ml}$ $(P<0.001)$ and $16.5 \pm 1.3 \mathrm{ml}(P<0.001)$ at 18 months,
$33.4 \pm 1.7 \mathrm{ml}(P<0.001)$ and $18.8 \pm 1.3 \mathrm{ml}(P<0.001)$ at 24 months, and $33.1 \pm 2.3 \mathrm{ml}(P<0.001)$ and $19.3 \pm 1.6$ $\mathrm{ml}(P<0.001)$ at 30 months after the surgery (Fig. $4 \mathrm{a}$ and b). There were no intragroup differences in the LAEF at 1 or 6 months after the operation $(P=0.166, P=0.053)$. The LAEF began to decrease from 12 months after the operation, and significant intragroup differences in the LAEF were observed at 12, 18, 24, and 30 months after the operation ( $P=0.041, P=0.003, P=0.002, P=0.002)$ in the experimental group (Fig. 4c).

Left ventricular volume and ejection fraction: Twoway ANOVA with repeated measures found no significant intergroup, intragroup or interactional differences in the LVEDV and LVESV. There were significant intergroup and intragroup differences for the LVEF, but no interactional difference (Table 1).

An independent-samples $t$-test found no intergroup differences in the LVEDV, LVESV, or LVEF before the operation ( $P=0.077, P=0.055, P=0.075$, respectively). There were no differences in the LVEDV between the two groups in the early post operation $(P=0.140$, $P=0.164, P=0.058, P=0.082$ at $1,6,12,18$ months after the operation, respectively), but in the late post operation, the LVEDV of the experimental group was higher than that in the control group ( $P=0.012, P=0.044$ at 24 and 30 months after the operation) (Table 1). No intergroup difference was found in LVESV at any time point after the operation. A significant intergroup difference was observed in the LVEF at 1, 6, 18, and 24 months after the operation $(P<0.001, P=0.017, P=0.002$, 
Table 1. Echocardiographic measurements in the control group and the MR group at different time points before and after the operation, as well as the results of the two-way repeated measures ANOVA with group (Control and MR) and time (different time points before and after the operation) as factors. Also shown are the results of an intergroup comparison using an independent-samples $t$-test and intragroup comparisons using a paired-samples $t$-test.

\begin{tabular}{|c|c|c|c|c|c|c|c|c|c|c|c|c|}
\hline & \multicolumn{2}{|c|}{ LAV-max } & \multicolumn{2}{|c|}{ LAV-min } & \multicolumn{2}{|c|}{ LAEF } & \multicolumn{2}{|c|}{ LVEDV } & \multicolumn{2}{|c|}{ LVESV } & \multicolumn{2}{|c|}{ LVEF } \\
\hline & $\mathrm{CON}$ & MR & $\mathrm{CON}$ & MR & $\mathrm{CON}$ & MR & $\mathrm{CON}$ & MR & $\mathrm{CON}$ & MR & $\mathrm{CON}$ & MR \\
\hline BS & $15.5 \pm 1.4$ & $12.1 \pm 1.5$ & $6.0 \pm 0.6$ & $4.8 \pm 0.5$ & $61.2 \pm 2.4$ & $60.4 \pm 2.1$ & $32.5 \pm 1.8$ & $26.8 \pm 2.2$ & $12.2 \pm 0.7$ & $7.8 \pm 1.0$ & $62.5 \pm 1.4$ & $68.8 \pm 2.6$ \\
\hline AS 1 month & $6.3 \pm 1.2$ & $5.8 \pm 1.9$ & $6.3 \pm 0.6$ & $11.5 \pm 1.2$ & $60.8 \pm 3.8$ & $54.2 \pm$ & $32.5 \pm 1.2$ & $38.4 \pm$ & $11.8 \pm 0.9$ & & $63.7 \pm 2.4$ & \\
\hline AS 6 month & $16.0 \pm 0.7$ & $31.0 \pm 1.9$ & $5.7 \pm 0.3$ & $14.9 \pm 1.4$ & $64.7 \pm 1.6$ & $51.3 \pm 2.9$ & $32.6 \pm 1.3$ & $36.6 \pm 2.1$ & $11.0 \pm 1.1$ & $9.4 \pm 0.9$ & $66.7 \pm 2.3$ & $74.6 \pm 1.8$ \\
\hline AS 12 month & $14.5 \pm 1.1$ & $30.0 \pm 2.0$ & $6.0 \pm 0.6$ & $15.6 \pm 1.3$ & $58.7 \pm 3.0$ & $47.3 \pm 4.4$ & $31.7 \pm 1.6$ & $37.6 \pm$ & $10.3 \pm 1.0$ & $10.0 \pm$ & $66.7 \pm 3.0$ & 73.8 \\
\hline AS 18 month & $15.0 \pm 1.2$ & $31.1 \pm 1.4$ & $5.7 \pm 0.6$ & $16.5 \pm 1.3$ & $62.0 \pm 2.7$ & $42.0 \pm 3.9$ & $32.7 \pm 0.7$ & $36.6 \pm$ & $12.3 \pm 0.6$ & $10.4 \pm 0.9$ & $61.7 \pm 1.2$ & $71.8=$ \\
\hline AS 24 month & $14.8 \pm 1.4$ & $33.4 \pm 1.7$ & $6.3 \pm 0.5$ & $18.8 \pm 1.3$ & $56.7 \pm 3.1$ & $43.6 \pm$ & $30.3 \pm 1.4$ & & $10.8 \pm 0.7$ & & $64.3 \pm 1.9$ & 1.6 \\
\hline $\begin{array}{l}\text { AS } 30 \text { month } \\
\text { Overall }\end{array}$ & $16.0 \pm 1.6$ & $33.1 \pm 2.3$ & $6.2 \pm 0.6$ & $19.3 \pm 1.6$ & $60.7 \pm 3.3$ & $41.2 \pm 3.1$ & $31.0 \pm 1.1$ & $37.4 \pm 2.3$ & $11.2 \pm 0.5$ & $11.9 \pm 1.3$ & $63.8 \pm 1.6$ & $68.6 \pm 2.1$ \\
\hline Inter & \multicolumn{2}{|c|}{$\mathrm{F}=36.108, P<0.001$} & \multicolumn{2}{|c|}{$\mathrm{F}=43.837, P<0.001$} & \multicolumn{2}{|c|}{$\mathrm{F}=13.510, P=0.003$} & \multicolumn{2}{|c|}{$\mathrm{F}=2.917, P=0.113$} & \multicolumn{2}{|c|}{$\mathrm{F}=1.150, P=0.305$} & \multicolumn{2}{|c|}{$\mathrm{F}=21.654, P=0.001$} \\
\hline Intragroup & \multicolumn{2}{|c|}{$\mathrm{F}=34.506, P<0.001$} & \multicolumn{2}{|c|}{$\mathrm{F}=27.157, P<0.001$} & \multicolumn{2}{|c|}{$\mathrm{F}=5.838, P=0.001$} & \multicolumn{2}{|c|}{$\mathrm{F}=5.581, P<0.001$} & \multicolumn{2}{|c|}{$\mathrm{F}=2.264, P=0.047$} & \multicolumn{2}{|c|}{$\mathrm{F}=3.280, P=0.007$} \\
\hline Interaction & \multicolumn{2}{|c|}{$\mathrm{F}=36.583, P<0.001$} & \multicolumn{2}{|c|}{$\mathrm{F}=26.854, P<0.001$} & \multicolumn{2}{|c|}{$\mathrm{F}=4.019, P=0.009$} & \multicolumn{2}{|c|}{$\mathrm{F}=7.725, P<0.001$} & \multicolumn{2}{|c|}{$\mathrm{F}=4.827, P<0.001$} & \multicolumn{2}{|c|}{$\mathrm{F}=1.833, P=0.105$} \\
\hline Intergroup & \multicolumn{2}{|c|}{ CON vs. MR } & \multicolumn{2}{|c|}{ CON vs. MR } & \multicolumn{2}{|c|}{ CON vs. MR } & \multicolumn{2}{|c|}{ CON vs. MR } & \multicolumn{2}{|c|}{ CON vs. MR } & $\mathrm{CON}$ & vs. MR \\
\hline BS & & .145 & & & & & $P=0$ & & & & & .075 \\
\hline AS1 month & $P=0$ & .002 & $P=$ & .005 & $P=0$ & .221 & $P=0$ & 140 & $P=0$ & & $P<0$ & 001 \\
\hline AS6 month & & 0.001 & & .001 & & .053 & $P=0$ & 164 & & & $P=0$ & 017 \\
\hline AS12 month & $P<0$ & 0.001 & $P<$ & .001 & $P=0$ & 0.070 & $P=0$ & .058 & $P=0$ & & $P=0$ & 102 \\
\hline AS18 month & $P<0$ & .001 & & & $P=0$ & .002 & $P=0$ & .082 & $P=0$ & & $P=0$ & 002 \\
\hline AS24 month & $P<0$ & 0.001 & $P<$ & .001 & $P=0$ & 0.006 & $P=0$ & .012 & $P=0$ & & $P=0$ & .032 \\
\hline AS30 month & $P<0$ & .001 & $P<$ & .001 & $P=0$ & .001 & $P=0$ & .044 & $P=0$ & & $P=0$ & .110 \\
\hline Intragroup & $\mathrm{CON}$ & MR & $\mathrm{CON}$ & MR & CON & MR & $\mathrm{CON}$ & MR & $\mathrm{CON}$ & MR & $\mathrm{CON}$ & MR \\
\hline AS $1 \mathrm{~m}$ vs BS & $P=0.224$ & $P<0.001$ & $P=0.576$ & $P=0.004$ & $P=0.903$ & $P=0.166$ & $P=1.000$ & $P=0.009$ & $P=0.611$ & $P=0.276$ & $P=0.656$ & $P=0.005$ \\
\hline AS6m vs BS & $P=0.665$ & $P<0.001$ & $P=0.363$ & $P=0.001$ & $P=0.300$ & $P=0.053$ & $P=0.915$ & $P=0.005$ & $P=0.158$ & $P=0.082$ & $P=0.097$ & $P=0.019$ \\
\hline AS $12 m$ vs BS & $P=0.203$ & $P<0.001$ & $P=1.000$ & $P<0.001$ & $P=0.542$ & $P=0.041$ & $P=0.659$ & $P=0.001$ & $P=0.058$ & $P=0.069$ & $P=0.351$ & $P=0.104$ \\
\hline AS $8 \mathrm{~m}$ vs BS & $P=0.542$ & $P<0.001$ & $P=0.576$ & $P<0.001$ & $P=0.843$ & $P=0.003$ & $P=0.913$ & $P=0.002$ & $P=0.862$ & $P=0.019$ & $P=0.714$ & $P=0.256$ \\
\hline AS $4 \mathrm{~m}$ vs BS & $P=0.416$ & $P<0.001$ & $P=0.638$ & $P<0.001$ & $P=0.349$ & $P=0.002$ & $P=0.311$ & $P=0.001$ & $P=0.082$ & $P=0.004$ & $P=0.396$ & $P=0.612$ \\
\hline AS30m vs BS & $P=0.597$ & $P<0.001$ & $P=0.741$ & $P<0.001$ & $P=0.898$ & $P=0.002$ & $P=0.357$ & $P=0.005$ & $P=0.076$ & $P=0.008$ & $P=0.387$ & $P=0.957$ \\
\hline
\end{tabular}

Data are means \pm SEM, CON: control, BS: before surgery, AS: after surgery, m: month.

$P=0.032$, respectively), but no intergroup differences were detected at 12 or 30 months after the operation ( $P=0.102, P=0.110$, respectively) (Table 1 ).

In the control group, there were no intragroup differences in the LVEDV, LVESV, or LVEF. In the experimental group, the LVEDV at all time points after the operation was higher than that before the operation (Fig. $4 d)$. There were no intragroup differences in the LVESV at 1,6 , or 12 months after the operation $(P=0.276$, $P=0.082, P=0.069$, respectively). The LVESV began to increase from 18 months after the operation, and a significant intragroup difference in LVESV was observed at 18,24 , and 30 months after the operation in the experimental group $(P=0.019, P=0.004, P=0.008$, respectively) (Fig. 4e). In the experimental group, the LVEF increased from $68.8 \pm 2.6 \%$ before the surgery to 78.8 $\pm 1.4 \%$ and $74.6 \pm 1.8 \%$ at 1 and 6 months after the surgery, respectively $(P=0.005, P=0.019$, respectively), after which it gradually decreased to near its preoperative level (Fig. 4f).

\section{D electrophysiological mapping}

There was no significant change in left atrial electrophysiological mapping. The low-voltage zone was not found in either the experimental or control groups in the three-dimensional mapping examination (Fig. 5).

\section{Histopathology findings}

Sirius Red staining showed that the CVF of the left atrium was $18.1 \pm 3.0 \%$ in the experimental group (Fig. $6 \mathrm{~b}$ and $\mathrm{f}$ ) and $1.6 \pm 0.2 \%$ in the control group (Fig. $6 \mathrm{a}$ and e). There was a significant difference between the two groups $(P<0.05)$. This difference was due to reactive fibrosis, manifesting as an increase in collagen deposition in the myocardial interstitial space, rather than reparative fibrosis. Sirius Red staining not only showed fibrosis but also that the fibrosis formation was due to the deposition of types I and III collagen. However, the CVF of the left ventricle was $2.0 \pm 0.5 \%$ in the experimental group (Fig. $6 \mathrm{~d}$ and $\mathrm{h}$ ) and $1.9 \pm 0.3 \%$ in the control group (Fig. 6c and g), with no significant difference between the two groups $(P=0.58)$. 

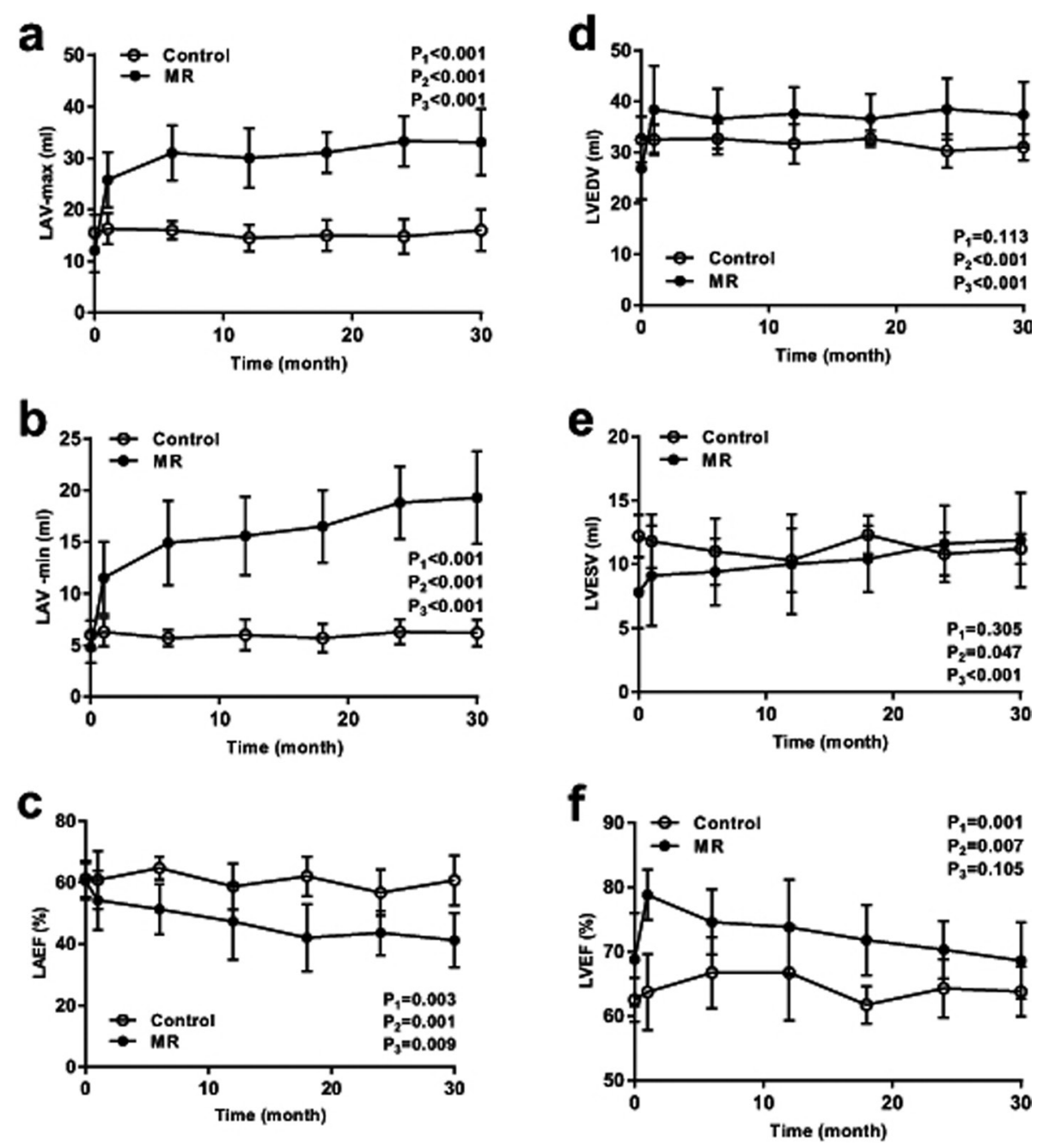

Fig. 4. Volume and systolic function of the left atrium and left ventricle. Comparison of LAV max (a), LAV min (b), LAEF (c), LVEDV (d), LVESV (e) and LVEF (f) between the experimental group and the control group. P1: $P$ value of intergroup comparison, P2: $P$ value of intragroup comparison, P3: $P$ value of interaction effect.

\section{Atrial fibrillation susceptibility test}

In the open-chest follow-up experiments, where atrial fibrillation susceptibility was tested by burst pacing, sustained atrial fibrillation was not observed in any of the 6 control pigs. In the MR group, sustained AF was inducible in 4 of 8 pigs (Fig. 7). There was a significant difference in atrial fibrillation susceptibility between the two groups $(\chi 2, P<0.05)$. The duration of atrial fibrillation in the experimental group was $310.9 \pm 103.8 \mathrm{~s}$, and the duration of atrial fibrillation in the control group was $19.5 \pm 4.4 \mathrm{~s}$.

Discussion

In this study, a porcine MR model with good uniformity and low mortality was established. During the process of model development, a standardized operative technique and the postoperative drug treatment regimen were developed. The operation was performed with a left chest incision, which protected the integrity of the chest more effectively than a median incision. We used epicardial ultrasonography during the operation, which provided clearer images than transesophageal echocardiography because of the large gap between the oesophagus and the heart in pigs. In addition, the oesophageal ultrasound probe was used instead of the conventional 

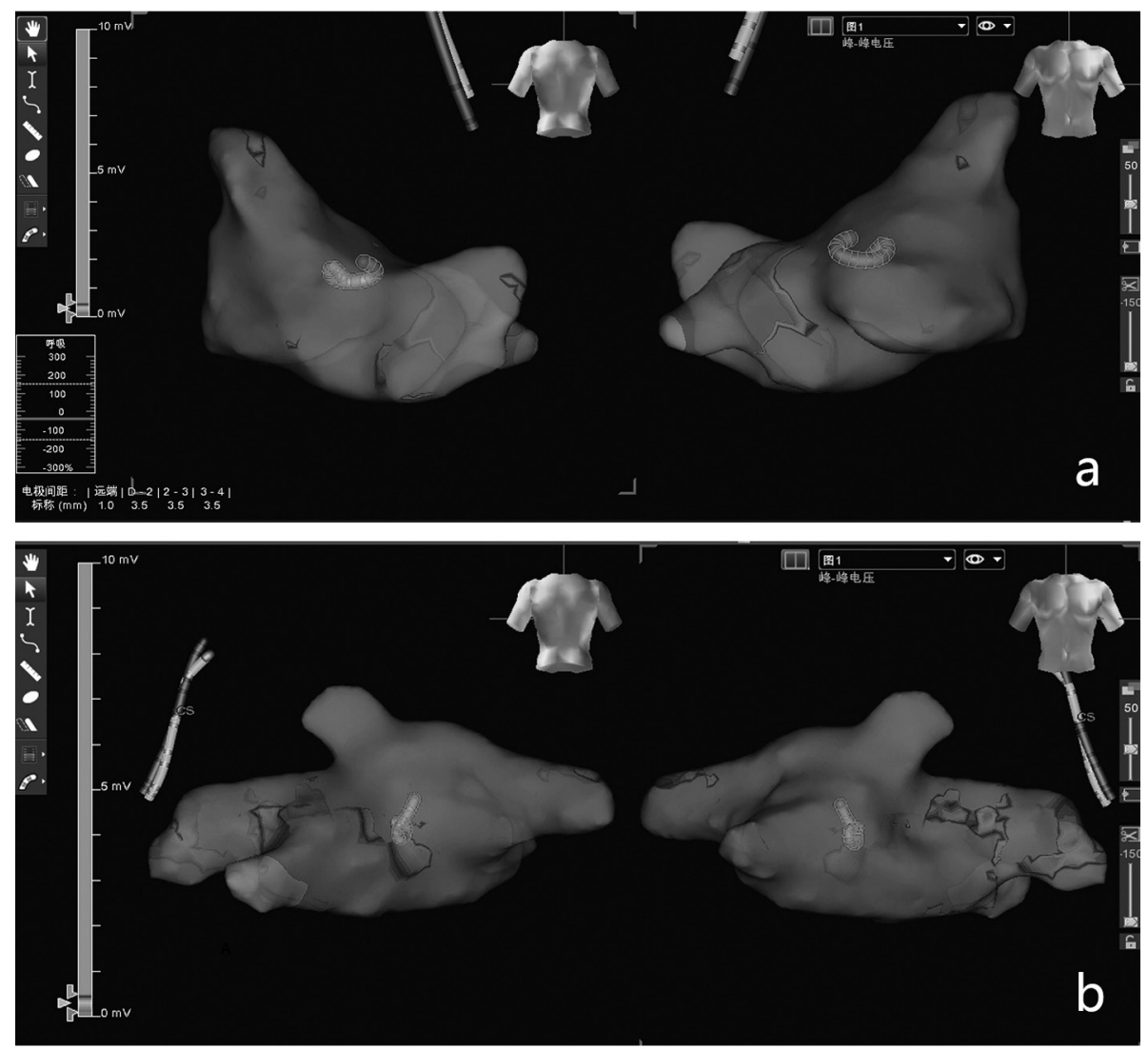

Fig. 5. The voltage map in the mitral regurgitation group and the normal group. Three-dimensional mapping showing no low-voltage zones in the left atrium of the control group (a) or the mitral regurgitation group (b).
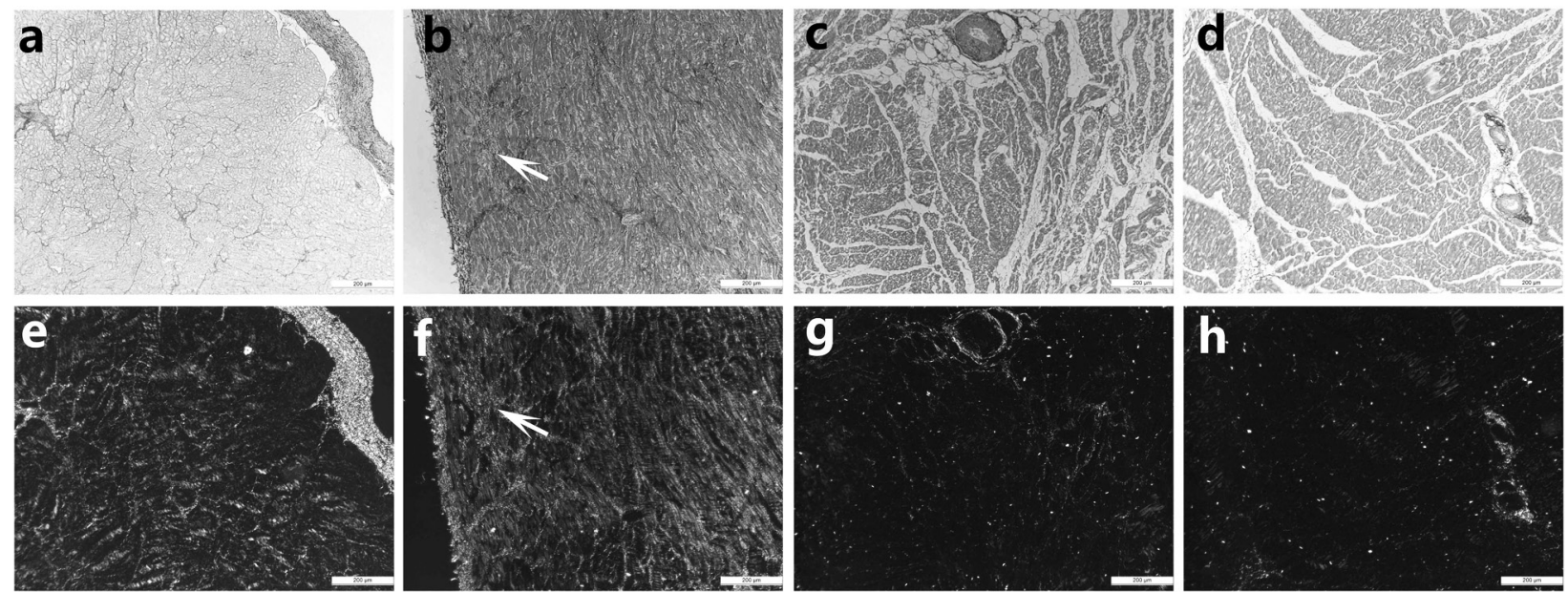

Fig. 6. The degree of fibrosis in the left atrium and left ventricle between the normal and mitral regurgitation groups. The left atria in the control group were stained with Sirius Red and then observed using light (a) and polarized light microscopy (e). The left atria in the MR group were stained with Sirius Red and observed using light (b) and polarized light microscopy (f). The left ventricles in the control group were stained with Sirius Red and observed using light (c) and polarized light microscopy (g). The left ventricle in the control group were stained with Sirius Red and observed by ordinary light microscopy (d) and polarized light microscopy (h). The arrow indicates the fibrotic region. 
a

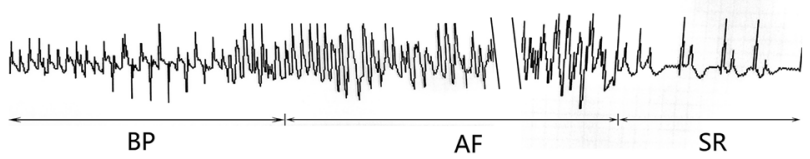

BP

b

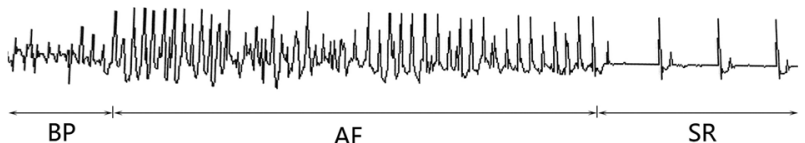

AF

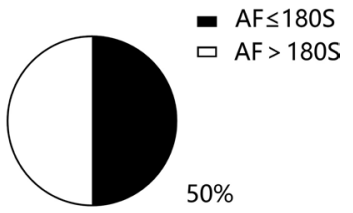

$50 \%$

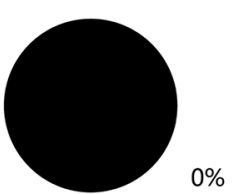

Fig. 7. ECG of the atrial fibrillation susceptibility test. When atrial fibrillation susceptibility tests were performed, the right atrial induction electrode recorded the ECG. BP: burst pacing; AF: atrial fibrillation; SR: sinus rhythm; the symbol "II" indicated that the middle part of AF was omitted. The positive rate of atrial fibrillation susceptibility was $50 \%$ in the mitral regurgitation group (a) and $0 \%$ in the normal group (b).

probe to minimize interference with the surgical procedures. Monitoring of the left atrial pressure can help to judge the degree of mitral regurgitation and to locate the mitral valve plane based on the change in pressure. However, a small challenge was encountered when the retractor entered the right ventricle to grasp chordae. Specifically, the retractor faced the atrial surface of the mitral valve, and the tendon was on the ventricular surface of the mitral valve, making it difficult for the retractor to grasp the tendon. Consequently, the surgeon had to turn the retractor to grasp the tendon, which was a simple task after a few attempts. During the course of the operation, the angle of the sheath tube should be kept unchanged or the retractor can move into the sheath in the wrong direction and damage the posterior wall of the atrium. One pig in the experiment died as a result of this complication. Postoperative short-term anti-infection and pain treatment is necessary. Anti-heart failure treatment for approximately 4 weeks after the operation can cause the induced acute MR flow to become chronic.

In this model, follow-up was performed for 30 months after the operation. The volume and systolic function of the left atrium and left ventricle were continuously observed. These metrics are considered the earliest affected part of the heart during MR. Therefore, the pathological process of the model was similar to the clinical course of chronic MR. Left atrial enlargement was evident, while the left ventricular volume and systolic function were preserved. This phenomenon may have occurred because during early MR, the left ventricle can pump excess blood into the low-pressure left atrium to avoid damage, resulting in enlargement of the left atrium due to the increased pressure and volume load. This condition differs from that of aortic regurgitation, in which the left ventricle must pump excess blood into the high-pressure aortic cavity and was damaged in the early stage. A similar result was obtained by histopathological examination. Severe fibrosis in the left atrium occurred in the experimental group compared with the control group, but there was no difference in left ventricular fibrosis between the two groups. Lastly, the results of three-dimensional mapping and atrial fibrillation susceptibility test suggests that although atrial fibrosis is not sufficient to alter the atrial potential, it can impact on electrical activity.

\section{Comparison with other MR models}

Organic MR model: Animal MR models are an important tool for cardiovascular research and can be implemented in large mammals, such as pigs [1, 14], dogs $[26,38]$, sheep $[16,34]$, as well as in small mammals, such as rabbits [11], rats [15, 27], among others. The rat MR model is produced via by thoracotomy. Under transoesophageal echocardiography, a needle is used to enter the ventricle through the apex and damage the mitral valve. The degree of regurgitation is generally evaluated by the ratio of the regurgitant beam area to the area of the left atrium, with a ratio of $45 \%$ considered appropriate. The 5-month survival rate is close to $60 \%$ [27]. The rabbit MR model can be induced via the left atrial appendage, and the success rate is relatively high [17]. Small animals have the advantage of low costs. In addition, genetically modified animals, an important tool in current scientific research, are easier to generate in 
small animals. However, their disadvantages are also clear. Small animals cannot be used for surgical repair or the evaluation of surgical methods and instruments. However, the evaluation of surgical procedures and instrumentation is an important aspect of MR model application.

Due to their size, MR in large animals can be produced using traditional thoracotomy and transcatheter techniques. Cutting off the mitral chordae or injuring the mitral valve leaflet are frequently used methods in largeanimal MR models. In a closed-chest model, the catheter can be inserted through the carotid $[14,20]$ or femoral artery [40]. The surgical procedure can be performed under fluoroscopic $[14,31]$ and/or transthoracic $[31,36]$ or transesophageal [40] Doppler echocardiographic guidance. MR is usually considered sufficiently severe when the cardiac output is decreased by $50 \%$ [ 6 , $20]$ or the pulmonary capillary wedge pressure is increased by $20 \mathrm{mmHg}[20,22]$ as these values are readily achievable during interventional procedures. One advantage of such models is the minimal invasiveness of the procedure. However, the location of the chordae and leaflet are difficult to determine, which may be an important reason for the poor uniformity of such models. Some animals must be excluded because the MR is only mild [17]. In an open-chest model, the operation can be completed either by left thoracotomy [2] or by median thoracotomy [1]. Mitral valve damage can be completed by atriotomy or using a metal device inserted though the LV apex [1] or auricula sinistra [2]. An atriotomy requires a cardiopulmonary bypass and is usually associated with cardiac arrest $[2,16]$, which increases the difficulty of surgery. However, the chordae can be cut under direct vision. However, it is impossible to estimate the degree of reflux under real-time ultrasonic monitoring. In the latter method, resection of the chordae is blind, but the accuracy is superior to that of closed-chest surgery because of the short operative path. Moreover, the procedure can be performed repeatedly under ultrasonic real-time monitoring until the degree of reflux is sufficient. In addition, the left atrial appendage pathway introduces less cardiac damage than the apical pathway. The mortality and complication rate of the operation vary widely in different reports. For example, mortality has been reported to be as high as $64 \%$ [17]. In contrast, complications are related to the operative technique. In the closed-chest model, the most common complication is congestive heart failure, with an incidence of up to
$41 \%$ [17]. In the open-chest model, the most common complication is pulmonary oedema [2], which may be related to the use of cardiopulmonary bypass or destruction of the integrity of the chest. In our study, there were few postoperative complications, possibly due to a small thoracic wound and a minor heart injury.

Functional MR model: Functional MR caused by myocardial ischaemia after the occlusion of coronary flow is a frequently used method of model establishment. Myocardial infarction in the posterior papillary muscle region can lead to displacement of the papillary muscles, which can itself cause mitral regurgitation. The target vessels for ischaemic mitral regurgitation are OM1, OM2, OM3, or OM4 in the left circumflex coronary artery [33], in some cases including the posterior descending branch of the right coronary artery [19]. This process can be completed by injecting procoagulant material into the target vessel under intervention [33] or ligating the coronary artery [19]. Because of the variation of the coronary artery distribution, the myocardial infarction area is uneven after the blood flow is interrupted. Consequently, the degree of papillary muscle displacement is variable and the degree of MR is not uniform. Another problem is that the degree of regurgitation of such models is generally mild. If greater MR is desired, Cui Yongchun et al. [7] reported that MR and myocardial ischaemia can both be achieved. However, only moderate MR was observed in their study. Greater reflux, associated with myocardial infarction, can lead to increased mortality. In the present study, because of the absence of myocardial infarction, we were able to increase the degree of reflux without increasing operative mortality. A large reflux can accelerate the disease process and save time. In addition, this model includes factors related to functional MR. Enlargement of the left atrium could displace the posterior mitral annulus onto the crest of the left ventricle inlet, which can reduce the posterior leaflet area available for coaptation and tethering of the posterior leaflet by increasing the annulo-papillary muscle distance [8].

Ex vivo MR model: In recent years, ex vivo platforms have been developed to simulate valvular pathologies, including mitral, tricuspid, and aortic injuries. The ex vivo valvular pathology model is usually implemented in a passive beating heart platform [13, 18, 42]. The system has three main components: (1) a computercontrolled pulse duplicator connected to the heart sample, (2) a simulator of the hydraulic input impedance of 
the systemic circulation, and (3) a preload reservoir [18]. Several other design systems have also been applied [3, 39]. The methods used to achieve MR are different and primarily include mitral annular dilatation and papillary muscle transposition. In the study of Bhattacharya et al. [3], mitral annulus dilation was achieved by injecting phenol into the muscular mitral annulus with a needle. The toxic substance in phenol can damage the structure and function of muscles and expand the mitral annulus. Alternatively, in the study by Richards et al. [28], annular dilation was replicated by manually stretching the mitral annulus until a $25 \%$ increase in the anterior-posterior distance occurred. In the study by Jaworek et al. [13], the anterior and posterior mitral annuli were pulled by an external force to achieve mitral annular dilatation, and papillary muscles displacement was achieved by pulling the papillary muscle. These ex vivo models, characterized by normal intracardiac anatomical relationships, replicate the main effects of the MR on many levels (haemodynamic valve morphology and kinematics) in a highly repeatable and controllable manner. The model is economic and simple and can be used not only in the research and development of surgical instruments but also in the teaching and training of doctors. However, such models have the following disadvantages: (1) The overall condition of the disease is not represented, and the model cannot be used in pathophysiological studies because there is no cellular remodelling, no hormone secretion, and no nerve stimulation. (2) The integrity of the heart as a whole is not reflected, including unilateral heart perfusion. This difference results in a loss of the effect of the right cardiac chamber, the absence of pericardial constraints, non-physiological blood flow, and an absence of cardiac systolic and diastolic process. These weaknesses, which have a relatively large impact on the valve in such a model, were eliminated in the present model. (3) A single organ is unable to provide a complete evaluation of the entire surgical instrument, which can be performed only with a complete animal. In comparison to the ex vivo bench models, animal models also have inherent disadvantages. Among these are biological consumption, a high cost and their timeconsuming nature. Both in vitro and in vivo models are important complementary tools for disease research and the development of new technologies. For this reason, such models should support rather than replace one another. For example, in the field of device development, the in vitro model can be used in early stages of experi- mentation. When the instruments are more mature, experiments in animals can be performed. We strongly believe that the combination of in vitro and in vivo methods can best solve these problems.

\section{Application of the model}

Pathophysiological study of MR: MR is divided into organic MR and functional MR. The early stage of this model is organic MR because we destroyed the mitral chordae. However, over time, the left atrium increases markedly in size, which can cause functional MR, as previously described. In addition, MR can also be divided into acute MR and chronic MR. Our model, similar to most MR models, is suitable for the study of acute MR. According to clinical needs, chronic MR, which has a higher incidence, is the primary subject of research. Therefore, it is very important that an acute MR model transitions into a chronic model. Medication is needed to gradually adapt the experimental animals to this change in haemodynamics. As noted above, postoperative drug therapy is the key to converting mitral valve acute MR into chronic MR.

Development and preclinical evaluation of surgical instruments: Over the past ten years, a wide range of devices and techniques for mitral valve repair have appeared, which can be used in percutaneous and openchest approaches. Research and development of these instruments have been carried out using MR models. Our model consists of tendon rupture, annulus dilatation, and left atrial enlargement, which are inherent in MR and are also required for the development of devices. In addition, an increasing number of cardiac electrophysiological tools have been applied clinically, such as threedimensional mapping system and radiofrequency ablation systems. The operation of these devices requires the heart cavity of the animal to be sufficiently large, especially the left atrium, to facilitate atrial septal puncture and operations in the left atrium. Our experiments show that the left atrium of a MR model pig becomes sufficiently large 6 months after surgery, meeting the needs of such experiments, and does not require excessive time or costs. Extending the time provides no benefit because the left atrial enlargement slows at later time points. In our experiments, three-dimensional mapping was also involved, and the surgeons believed that the model group was more similar to the clinical condition than the normal pigs.

Myocardial fibrosis: Myocardial fibrosis is an impor- 
tant aspect of the study of heart diseases. At present, the commonly used models of myocardial fibrosis are primarily established by increasing the pressure load [30], immune damage [25], ischaemia [5] and high glucose [43]. However, none of these models was specific to atrial fibrosis, and MR is an ideal model for studying this condition. However, the porcine MR model is not a good option for the study of ventricular fibrosis because the duration of the present experiment was 30 months, during which the left ventricle, as an affected organ, became less fibrotic.

Atrial fibrillation: The porcine MR model cannot be used for the study of spontaneous atrial fibrillation, which we studied for 30 months without spontaneous atrial fibrillation in any cases. According to the result of three-dimensional mapping, no low-voltage zones, which are thought to be associated with atrial fibrillation, were found in either the experimental or control groups. Atrial fibrillation has a complex mechanism [12], and potentially because of differences in genetic background or the structure of the heart, it is difficult for pigs to achieve spontaneous atrial fibrillation by MR. Additionally, there are no previous reports of large-animal models of spontaneous atrial fibrillation. Our experiments showed that MR was associated with increased susceptibility to atrial fibrillation, and in the presence of the same electrical stimuli, atrial fibrillation was more likely to occur in the experimental group. These findings are consistent with previous results [40]. If pacemakers are used simultaneously, MR can be used as a tool to study atrial fibrillation. Previous studies have shown that combined MR and atrial high-frequency stimulation allow for a longer maintenance of the animal independently of atrial fibrillation [10].

Heart failure: The porcine MR model can be used to study acute heart failure in that MR can result in a sudden change in haemodynamics. The heart fails to adapt to this change, causing acute heart failure. Previous reports have described cases of postoperative death from heart failure [17], and the establishment of a heart failure model in this manner has been reported [32]. Postoperative medication is an effective way to prevent animals from dying from acute heart failure, as shown previously. We conducted a 30-month observational study of MR in pigs and conclude that it is not suitable for studies of chronic heart failure. Once the animals passed through acute MR, they were able to maintain smooth heart function for a long period of time without heart failure. Therefore, we do not believe that MR is suitable for establishing a chronic heart failure model because it may take a longer time for heart failure to develop and may associated with excessive time and costs.

\section{Conclusion}

Our method provides a simple and effective way to generate a MR model with severe reflux. With the monitoring by epicardial ultrasound and left atrial pressure, the survival rate and success rate of the operation is encouraging. The treatment of the postoperative animal model allowed for a smooth transition from the acute to the chronic period, which is a critical step. In addition to pathophysiological studies of MR, this model can be used for the development and preclinical evaluation of surgical instruments, atrial fibrillation, myocardial fibrosis and acute heart failure, among other applications. However, this model sis not suitable for studies of chronic heart failure.

\section{Conflict of Interest}

The authors declare that they have no competing interests.

\section{Acknowledgments}

This work was supported by the National Natural Science Foundation of China (Project No: 81370190).

\section{References}

1. Bajona, P., Katz, W.E., Daly, R.C., Zehr, K.J., and Speziali, G. 2009. Beating-heart, off-pump mitral valve repair by implantation of artificial chordae tendineae: an acute in vivo animal study. J. Thorac. Cardiovasc. Surg. 137: 188-193. [Medline] [CrossRef]

2. Bernal, J.M., García, I., Morales, D., Diago, C., Ruiz, B., Val, F., Ojeda, J.L., and Revuelta, J.M. 2006. The 'Valve Racket': a new and different concept of atrioventricular valve repair. Eur. J. Cardiothorac. Surg. 29: 1026-1029. [Medline] [CrossRef]

3. Bhattacharya, S., Pham, T., He, Z., and Sun, W. 2014. Tension to passively cinch the mitral annulus through coronary sinus access: an ex vivo study in ovine model. J. Biomech. 47: 1382-1388. [Medline] [CrossRef]

4. Borer, J.S. 2014. Early surgery or watchful waiting for asymptomatic severe degenerative mitral regurgitation: is the answer now clear? J. Am. Coll. Cardiol. 63: 2408-2410. [Medline] [CrossRef] 
5. Cardin, S., Guasch, E., Luo, X., Naud, P., Le Quang, K., Shi, Y., Tardif, J.C., Comtois, P., and Nattel, S. 2012. Role for MicroRNA-21 in atrial profibrillatory fibrotic remodeling associated with experimental postinfarction heart failure. Circ. Arrhythm. Electrophysiol. 5: 1027-1035. [Medline] [CrossRef]

6. Choi, H., Lee, K., Lee, H., Lee, Y., Chang, D., Eom, K., Youn, H., Choi, M., and Yoon, J. 2004. Quantification of mitral regurgitation using proximal isovelocity surface area method in dogs. J. Vet. Sci. 5: 163-171. [Medline]

7. Cui, Y.C., Li, K., Tian, Y., Yuan, W.M., Peng, P., Yang, J.Z., Zhang, B.J., Zhang, H.D., Wu, A.L., and Tang, Y. 2014. A pig model of ischemic mitral regurgitation induced by mitral chordae tendinae rupture and implantation of an ameroid constrictor. PLoS One 9: e111689. [Medline] [CrossRef]

8. Delgado, V. and Bax, J.J. 2017. Atrial Functional Mitral Regurgitation: From Mitral Annulus Dilatation to Insufficient Leaflet Remodeling. Circ. Cardiovasc. Imaging 10: e006239. [Medline] [CrossRef]

9. Duerr, G.D., Sinning, J.M., and Mellert, F. 2016. New expandable mitral annuloplasty ring facilitates transcatheter mitral valve implantation: proof of concept. EuroIntervention 11: e1662-e1668. [Medline] [CrossRef]

10. Everett, T.H. 4th., Li, H., Mangrum, J.M., McRury, I.D., Mitchell, M.A., Redick, J.A., and Haines, D.E. 2000. Electrical, morphological, and ultrastructural remodeling and reverse remodeling in a canine model of chronic atrial fibrillation. Circulation 102: 1454-1460. [Medline] [CrossRef]

11. Gunawardena, S., Bravo, E., and Kappagoda, C.T. 1999. Rapidly adapting receptors in a rabbit model of mitral regurgitation. J. Physiol. 521: 739-748. [Medline] [CrossRef]

12. January, C.T., Wann, L.S., Alpert, J.S., Calkins, H., Cigarroa, J.E., Cleveland, J.C. Jr., Conti, J.B., Ellinor, P.T., Ezekowitz, M.D., Field, M.E., Murray, K.T., Sacco, R.L., Stevenson, W.G., Tchou, P.J., Tracy, C.M., Yancy, C.W., American College of Cardiology/American Heart Association Task Force on Practice Guidelines 2014. 2014 AHA/ACC/HRS guideline for the management of patients with atrial fibrillation: a report of the American College of Cardiology/American Heart Association Task Force on Practice Guidelines and the Heart Rhythm Society. J. Am. Coll. Cardiol. 64: e1-e76. [Medline] [CrossRef]

13. Jaworek, M., Lucherini, F., Romagnoni, C., Gelpi, G., Contino, M., Romitelli, P., Antona, C., Fiore, G.B., and Vismara, R. 2017. Modelling of Lesions Associated with Functional Mitral Regurgitation in an Ex Vivo Platform. Ann. Biomed. Eng. 45: 2324-2334. [Medline] [CrossRef]

14. Kawase, Y., Ly, H.Q., Prunier, F., Lebeche, D., Shi, Y., Jin, H., Hadri, L., Yoneyama, R., Hoshino, K., Takewa, Y., Sakata, S., Peluso, R., Zsebo, K., Gwathmey, J.K., Tardif, J.C., Tanguay, J.F., and Hajjar, R.J. 2008. Reversal of cardiac dysfunction after long-term expression of SERCA2a by gene transfer in a pre-clinical model of heart failure. J. Am. Coll. Cardiol. 51: 1112-1119. [Medline] [CrossRef]

15. Kim, K.H., Kim, Y.J., Ohn, J.H., Yang, J., Lee, S.E., Lee, S.W., Kim, H.K., Seo, J.W., and Sohn, D.W. 2012. Longterm effects of sildenafil in a rat model of chronic mitral regurgitation: benefits of ventricular remodeling and exercise capacity. Circulation 125: 1390-1401. [Medline] [CrossRef]

16. Kim, Y.J., Jones, M., Greenberg, N.L., Popovic, Z.B., Sitges, M., Bauer, F., Thomas, J.D., and Shiota, T. 2007. Evaluation of left ventricular contractile function using noninvasively determined single-beat end-systolic elastance in mitral regurgitation: experimental validation and clinical application. J. Am. Soc. Echocardiogr. 20: 1086-1092. [Medline] [CrossRef]

17. Kleaveland, J.P., Kussmaul, W.G., Vinciguerra, T., Diters, R., and Carabello, B.A. 1988. Volume overload hypertrophy in a closed-chest model of mitral regurgitation. Am. J. Physiol. 254: H1034-H1041. [Medline]

18. Leopaldi, A.M., Vismara, R., Lemma, M., Valerio, L., Cervo, M., Mangini, A., Contino, M., Redaelli, A., Antona, C., and Fiore, G.B. 2012. In vitro hemodynamics and valve imaging in passive beating hearts. J. Biomech. 45: 1133-1139. [Medline] [CrossRef]

19. Minakawa, M., Robb, J.D., Morital, M., Koomalsinghl, K.J., Vergnat, M., Gillespie, M.J., Gorman, J.H. 3rd., and Gorman, R.C. 2014. A model of ischemic mitral regurgitation in pigs with three-dimensional echocardiographic assessment. J. Heart Valve Dis. 23: 713-720. [Medline]

20. Monnet, E. and Chachques, J.C. 2005. Animal models of heart failure: what is new? Ann. Thorac. Surg. 79: 14451453. [Medline] [CrossRef]

21. Naito, N., Nishimura, T., Takewa, Y., Kishimoto, S., Date, K., Umeki, A., Ando, M., Ono, M., and Tatsumi, E. 2016. What Is the Optimal Setting for a Continuous-Flow Left Ventricular Assist Device in Severe Mitral Regurgitation? Artif. Organs 40: 1039-1045. [Medline] [CrossRef]

22. Nemoto, S., Hamawaki, M., De Freitas, G., and Carabello, B.A. 2002. Differential effects of the angiotensin-converting enzyme inhibitor lisinopril versus the beta-adrenergic receptor blocker atenolol on hemodynamics and left ventricular contractile function in experimental mitral regurgitation. $J$. Am. Coll. Cardiol. 40: 149-154. [Medline] [CrossRef]

23. Nishimura, R.A., Otto, C.M., Bonow, R.O., Carabello, B.A., Erwin, J.P. 3rd., Guyton, R.A., O'Gara, P.T., Ruiz, C.E., Skubas, N.J., Sorajja, P., Sundt, T.M. 3rd., Thomas, J.D., ACC/AHA Task Force Members 2014. 2014 AHA/ACC Guideline for the Management of Patients With Valvular Heart Disease: a report of the American College of Cardiology/American Heart Association Task Force on Practice Guidelines. Circulation 129: e521-e643. [Medline] [CrossRef]

24. Nkomo, V.T., Gardin, J.M., Skelton, T.N., Gottdiener, J.S., Scott, C.G., and Enriquez-Sarano, M. 2006. Burden of valvular heart diseases: a population-based study. Lancet 368 : 1005-1011. [Medline] [CrossRef]

25. Ohshima, M., Yamahara, K., Ishikane, S., Harada, K., Tsuda, H., Otani, K., Taguchi, A., Miyazato, M., Katsuragi, S., Yoshimatsu, J., Kodama, M., Kangawa, K., and Ikeda, T. 2012. Systemic transplantation of allogenic fetal membranederived mesenchymal stem cells suppresses Th1 and Th17 T cell responses in experimental autoimmune myocarditis. $J$. Mol. Cell. Cardiol. 53: 420-428. [Medline] [CrossRef]

26. Perry, G.J., Wei, C.C., Hankes, G.H., Dillon, S.R., Rynders, 
P., Mukherjee, R., Spinale, F.G., and Dell'Italia, L.J. 2002. Angiotensin II receptor blockade does not improve left ventricular function and remodeling in subacute mitral regurgitation in the dog. J. Am. Coll. Cardiol. 39: 1374-1379. [Medline] [CrossRef]

27. Pu, M., Gao, Z., Li, J., Sinoway, L., and Davidson, W.R. Jr. 2005. Development of a new animal model of chronic mitral regurgitation in rats under transesophageal echocardiographic guidance. J. Am. Soc. Echocardiogr. 18: 468-474. [Medline] [CrossRef]

28. Richards, A.L., Cook, R.C., Bolotin, G., and Buckner, G.D. 2009. A dynamic heart system to facilitate the development of mitral valve repair techniques. Ann. Biomed. Eng. 37: 651-660. [Medline] [CrossRef]

29. Ring, L., Rana, B.S., Wells, F.C., Kydd, A.C., and Dutka, D.P. 2014. Atrial function as a guide to timing of intervention in mitral valve prolapse with mitral regurgitation. JACC Cardiovasc. Imaging 7: 225-232. [Medline] [CrossRef]

30. Rizzi, E., Ceron, C.S., Guimaraes, D.A., Prado, C.M., Rossi, M.A., Gerlach, R.F., and Tanus-Santos, J.E. 2013. Temporal changes in cardiac matrix metalloproteinase activity, oxidative stress, and TGF- $\beta$ in renovascular hypertension-induced cardiac hypertrophy. Exp. Mol. Pathol. 94: 1-9. [Medline] [CrossRef]

31. Sabri, A., Rafiq, K., Seqqat, R., Kolpakov, M.A., Dillon, R., and Dell'italia, L.J. 2008. Sympathetic activation causes focal adhesion signaling alteration in early compensated volume overload attributable to isolated mitral regurgitation in the dog. Circ. Res. 102: 1127-1136. [Medline] [CrossRef]

32. Satoh, K., Masuda, T., Hasegawa, N., Marumo, F., and Kikawada, R. 1989. [The concentration of atrial natriuretic peptide (ANP) in plasma and atrial tissue of canines with acute heart failure induced by reversible aortic or mitral regurgitation]. Nippon Naibunpi Gakkai Zasshi 65: 794-806. (in Japanese) [Medline]

33. Shi, W., McIver, B.V., Kalra, K., Sarin, E.L., Schmarkey, S., Duggan, M., Thourani, V.H., Guyton, R.A., and Padala, M. 2017. A Swine Model of Percutaneous Intracoronary Ethanol Induced Acute Myocardial Infarction and Ischemic Mitral Regurgitation. J. Cardiovasc. Transl. Res. 10: 391-400. [Medline] [CrossRef]

34. Shiota, T., Jones, M., Teien, D.E., Yamada, I., Passafini, A., Ge, S., and Sahn, D.J. 1995. Dynamic change in mitral regurgitant orifice area: comparison of color Doppler echocardiographic and electromagnetic flowmeter-based methods in a chronic animal model. J. Am. Coll. Cardiol. 26: 528-536. [Medline] [CrossRef]

35. Sondergaard, L., Ussia, G.P., Dumonteil, N. and Quadri, A. 2015. The CardiAQ transcatheter mitral valve implantation system. EuroIntervention 11 Suppl W: W76-77.

36. Sun, Q., Tang, M., Pu, J., and Zhang, S. 2008. Pulmonary venous structural remodeling in a canine model of chronic atrial dilation due to mitral regurgitation. Can. J. Cardiol. 24: 305-308. [Medline] [CrossRef]

37. Suri, R.M., Vanoverschelde, J.L., Grigioni, F., Schaff, H.V., Tribouilloy, C., Avierinos, J.F., Barbieri, A., Pasquet, A., Huebner, M., Rusinaru, D., Russo, A., Michelena, H.I., and Enriquez-Sarano, M. 2013. Association between early surgical intervention vs watchful waiting and outcomes for mitral regurgitation due to flail mitral valve leaflets. JAMA 310 : 609-616. [Medline] [CrossRef]

38. Tallaj, J., Wei, C.C., Hankes, G.H., Holland, M., Rynders, P., Dillon, A.R., Ardell, J.L., Armour, J.A., Lucchesi, P.A., and Dell'Italia, L.J. 2003. Beta1-adrenergic receptor blockade attenuates angiotensin II-mediated catecholamine release into the cardiac interstitium in mitral regurgitation. Circulation 108: 225-230. [Medline] [CrossRef]

39. Taramasso, M., Emmert, M.Y., Reser, D., Guidotti, A., Cesarovic, N., Campagnol, M., Addis, A., Nietlispach, F., Hoerstrup, S.P., and Maisano, F. 2015. Pre-clinical In Vitro and In Vivo Models for Heart Valve Therapies. J. Cardiovasc. Transl. Res. 8: 319-327. [Medline] [CrossRef]

40. Verheule, S., Wilson, E., Everett, T. 4th., Shanbhag, S., Golden, C., and Olgin, J. 2003. Alterations in atrial electrophysiology and tissue structure in a canine model of chronic atrial dilatation due to mitral regurgitation. Circulation 107: 2615-2622. [Medline]

41. Verheye, S., Cheung, A., Leon, M. and Banai, S. 2015. The Tiara transcatheter mitral valve implantation system. EuroIntervention 11 Suppl W: W71-72.

42. Vismara, R., Leopaldi, A.M., Piola, M., Asselta, C., Lemma, M., Antona, C., Redaelli, A., van de Vosse, F., Rutten, M., and Fiore, G.B. 2016. In vitro assessment of mitral valve function in cyclically pressurized porcine hearts. Med. Eng. Phys. 38: 346-353. [Medline] [CrossRef]

43. Zhang, D., Cui, Y., Li, B., Luo, X., Li, B., and Tang, Y. 2016. miR-155 regulates high glucose-induced cardiac fibrosis via the TGF- $\beta$ signaling pathway. Mol. Biosyst. 13: 215-224. [Medline] [CrossRef] 